\title{
Cardiovascular Morbidity and Mortality in Rheumatoid Arthritis
}

\author{
Sherine E. Gabriel, MD \\ Department of Health Sciences Research, Mayo Clinic, Rochester, MN
}

\section{Abstract}

Patients with rheumatoid arthritis (RA) are at increased risk of mortality compared with the general population. Evidence suggests that this increased mortality can largely be attributed to increased cardiovascular $(\mathrm{CV})$ death. In a retrospective study of an inception cohort of RA patients in Rochester, MN, we found that patients with RA were at increased risk of CV death, ischemic heart disease, and heart failure compared with age- and sex-matched community controls. In addition, when we examined coronary artery tissue from autopsied RA patients, we observed increased evidence of inflammation and an increased proportion of unstable plaques. We also investigated the contribution of traditional and RA-specific risk factors to this increased risk of $\mathrm{CV}$ morbidity and mortality. Although traditional CV disease risk factors were found to contribute to the increased risk of mortality in RA patients, they did not fully explain the increased CV mortality observed in RA. Instead, increased inflammation associated with RA appears to contribute substantially to the increased CV mortality. Together with other studies that have demonstrated similar associations between RA and CV mortality, these data suggest that more aggressive management of inflammation in RA may lead to significant improvements in outcomes for patients with RA.

\section{Keywords}

Rheumatoid arthritis; ischemic heart disease; congestive heart failure; epidemiology; cardiovascular mortality

\section{Introduction}

Many studies, dating back more than 50 years, have reported an increased risk of mortality
in patients with rheumatoid arthritis (RA). $1^{-} 5$ Recent evidence has demonstrated that this
increased risk may be largely attributable to cardiovascular $(\mathrm{CV})$ events. $6^{-} 9$ Although it has
been previously reported that $\mathrm{CV}$ morbidity and mortality are increased in RA, it has been
unclear whether the pattern of $\mathrm{CV}$ disease is similar in patients with and without RA.
Furthermore, the contribution of traditional risk factors, RA disease characteristics, and RA
treatments to this mortality has been uncertain. In particular, given the recent appreciation of
the important role of inflammatory processes in the development and progression of
atherosclerosis, 10 interest has focused on the CV risk that might be associated with systemic

(c) 2009 Elsevier Inc. All rights reserved.

Address correspondence to: Sherine E. Gabriel, MD, Professor of Medicine, Professor of Epidemiology, Mayo Clinic College of Medicine, Chair, Department of Health Sciences Research, Mayo Clinic, 200 First Street SW, Rochester, MN 55905, Phone: 507-284-1696, gabriel@mayo.edu.

Publisher's Disclaimer: This is a PDF file of an unedited manuscript that has been accepted for publication. As a service to our customers we are providing this early version of the manuscript. The manuscript will undergo copyediting, typesetting, and review of the resulting proof before it is published in its final citable form. Please note that during the production process errors may be discovered which could affect the content, and all legal disclaimers that apply to the journal pertain. 
inflammation in RA. This review will focus on studies investigating these questions conducted using the Rochester Epidemiology Project and will compare data obtained in these studies with other published literature. Additionally, the implications of these findings for the management of inflammation in RA will be discussed.

\section{Study Design}

The Rochester Epidemiology Project is a data resource that is ideally suited to the population-based investigation of long-term outcomes in RA. Its data linkage system provides access to the Mayo Clinic unified medical record system, and the complete (inpatient and outpatient) medical records of all healthcare providers in Olmsted County, $\mathrm{MN}$ are captured. ${ }^{11}$ Consequently, virtually every case of RA fulfilling American College of Rheumatology (ACR) diagnostic criteria in the local population is captured in this system.

To investigate these questions, we assembled an incidence cohort of approximately 600 RA patients who first met the ACR 1987 criteria for RA between January 1, 1955, and December 31, 1994. The incidence date of RA was defined as the date on which patients met 4 of the 7 ACR 1987 criteria. Additionally, we assembled a comparison cohort of nonRA controls who were matched based on their age, sex, and length of medical history. ${ }^{12} \mathrm{We}$ then followed these 2 cohorts retrospectively through their medical records over a long follow-up period (approximately $15 \mathrm{y}$ in each cohort), and detailed information regarding $\mathrm{RA}, \mathrm{CV}$ risk factors, and $\mathrm{CV}$ outcomes was collected. ${ }^{12,13}$

\section{Cardiovascular Disease Outcomes in Rheumatoid Arthritis}

\section{Cardiovascular Death}

Data from the Rochester Epidemiology Project have confirmed and extended previous studies that demonstrated an increased mortality in individuals with RA. Overall, patients in the RA cohort were found to have a markedly increased risk of death compared with the general population. Between 1955 and 1994, patients in the RA cohort had a significantly increased risk of death compared with age- and sex-matched controls $(P<0.001$; standardized mortality ratio [SMR], 1.27; 95\% confidence interval [CI], 1.13-1.41) (Figure 1). ${ }^{14}$ In this incidence cohort (of which $73 \%$ were female), women had a marked increase in mortality compared with men (SMR 1.41 in women vs 1.08 in men).

We (and others) have shown that the increased risk of mortality in the RA population is due, in large part, to an increased risk of CV death. In the Rochester RA cohort, 49.7\% (176 of 354) of deaths occurring during the study period were attributed to CV causes according to medical records and/or death certificates. ${ }^{15}$ Wallberg-Jonsson et al16 reported that among a cohort of 606 persons with RA, $53 \%$ of the 265 deaths during the study period were attributed to CV disease. In this study, compared with the general population, the SMR for $\mathrm{CV}$ death was $1.36(P<0.05)$ in men, whereas in women it was $1.54(P<0.001)$. Overall, the SMR for CV death was $1.46(P<0.001)$. In a cohort of 1010 RA patients, Goodson et al ${ }^{17}$ reported that the SMR for CV death in men was 1.36 (95\% CI, 1.04-1.75), whereas in women the SMR was 1.93 (1.65-2.26). This increase in the risk of CV death was more pronounced in rheumatoid factor-positive (RF+) patients: the SMR for CV death in seropositive women was 2.10 (95\% CI, 1.73-2.51) compared with 1.59 (95\% CI, 1.14-2.15) in seronegative women. In seropositive men, the SMR for CV death was 1.59 (95\% CI, 1.19-2.09) compared with 0.81 (95\% CI, 0.41-1.46) in seronegative men.

The mortality gap between RA patients and the general population is not closing. When patients in the Rochester RA cohort were grouped by the decade of RA incidence (ie, the decade in which they first met ACR 1987 criteria), no significant difference in survival was 
observed over the 4 decades of the study (Figure 2). ${ }^{14}$ Indeed, some evidence suggests that the mortality gap between persons with RA and the general population may be widening. Although the mortality rate in the RA cohort was found to remain relatively constant at 2.1 per 100 person-years between 1965 and 2000, the mortality rate of Minnesota's white population decreased substantially from 1.7 to 1.1 per 100 person-years over the same period. ${ }^{18}$ Consequently, the SMR increased from 1.2 in 1965 to 1.9 in $2000(P=0.024)$. A further comparison of mortality in the RA cohort and the general population suggested that this widening mortality gap was confined to the RF+ RA population. ${ }^{18}$

\section{Ischemic Heart Disease}

In subsequent studies, patients in the Rochester RA cohort were found to be at increased risk of ischemic heart disease (Table 1). ${ }^{9}$ In the 2-year period immediately preceding the fulfillment of the ACR 1987 criteria, RA patients were more likely to experience hospitalization for myocardial infarction (MI) (odds ratio [OR], 3.17; 95\% CI, 1.16-8.68) and unrecognized ("silent") MI (OR, 5.86; 95\% CI, 1.29-26.64) than age- and sex-matched controls. The increased risk of unrecognized MI persisted after the incidence of RA (hazard ratio [HR], 2.13; 95\% CI, 1.13-4.03). In addition, the risk of sudden death in RA also markedly increased after the index date (HR, 1.94; 95\% CI, 1.06-3.55) compared with ageand sex-matched controls.

Notably, patients in the RA cohort were less likely to experience angina pectoris either before (OR, 0.58; 95\% CI, 0.34-0.99) or after (HR, 0.76; 95\% CI, 0.52-1.13) the incidence date. The risk of angina pectoris after the incidence date was significantly lower in RA compared with non-RA patients, when patients with a history of hospitalized MI, unrecognized MI, or angina pectoris before the incidence date were excluded from the analysis $(P=0.025)$. The lower observed probability of angina pectoris in RA patients may have been because patients with RA were less likely to report chest pain or because they (or their physicians) failed to attribute it to cardiac origin.

Overall, these results are consistent with a number of previous studies that have demonstrated an increased risk of ischemic heart disease in patients with RA. ${ }^{1,8,19-23}$ Furthermore, these data demonstrate that the patterns of ischemic heart disease are markedly different in RA and non-RA patients. Specifically, in patients with RA, ischemic heart disease was more likely to remain silent and to manifest as sudden death. To further investigate this finding, a histological examination of coronary artery tissue from 41 autopsied RA patients and 82 age-and sex-matched controls was undertaken. ${ }^{24}$ Overall, the extent of coronary artery stenosis and the number of acute lesions were similar in the 2 groups. However, more inflammation of the left circumflex and left anterior descending coronary arteries was observed in RA patients than in controls (32\% vs $11 \%, P<0.005$ and $90 \%$ vs $72 \%, P=0.024$, respectively). More extensive differences in the pattern of coronary artery disease were observed when the subgroup of RA patients with preexisting coronary artery disease and their matched controls were compared. Compared with the control group, fewer RA patients had multiple vessel disease ( $32 \%$ vs $61 \%, P=0.018)$, and they had lesssevere coronary artery atherosclerosis $(32 \%$ had a score $\underline{U}$ compared with $61 \%$ of non-RA patients, $P=0.011$ ) and a lower grade of stenosis in the left main and left circumflex coronary arteries $(P=0.023$ and $P=0.029$, respectively). However, in RA patients, $48 \%$ of plaques in the left anterior descending coronary artery were classified as vulnerable (ie, unstable) compared with $22 \%$ in the non-RA patients $(P=0.018)$. Overall, these data suggest that the etiology of CV disease may be different in RA than in non-RA patients, with inflammation playing an even greater role in the development of disease. 


\section{Heart Failure}

Patients with RA are also at increased risk of heart failure compared with the general population. 25,26 In the Rochester RA cohort, the cumulative incidence of congestive heart failure (CHF; defined according to the Framingham criteria) at 30-year follow-up was 34\% compared with $25 \%$ in the non-RA cohort (Figure 3; $P<0.001$ ).27 Even after adjustment for $\mathrm{CV}$ risk factors and ischemic heart disease, in addition to demographics, patients with RA had almost twice the risk of developing CHF as non-RA patients (HR, 1.87; 95\% CI, 1.472.39). This increased risk of CHF appeared to be limited to the subgroup of RA patients who were RF+. The risk of CHF was significantly increased in the 374 seropositive patients in the RA cohort (HR, 2.59; 95\% CI, 1.95-3.43), but it was not significantly elevated in the 201 seronegative patients (HR, 1.28; 95\% CI, 0.93-1.78). In addition, a preliminary report indicated that patients with RA are more likely to have heart failure with preserved ejection fraction than non-RA patients (OR, 2.57; 95\% CI, 1.20-5.49). ${ }^{28}$

Patients with RA also appeared to have poorer outcomes after heart failure. Patients with RA were at increased risk for death in the period immediately after heart failure compared with non-RA patients. In the 30 days after heart failure, $16 \%$ of RA patients died, compared with $7 \%$ of non-RA patients $(P<0.001)$. The risk of death remained elevated 6 months after heart failure (OR, 1.94; 95\% CI, 1.17-3.23). ${ }^{29}$

\section{Risk Factors for Cardiovascular Disease in Rheumatoid Arthritis}

Though it is apparent that RA patients are at increased risk of developing CV disease and that this contributes to the increased mortality observed in the RA population, the factors responsible for this increased $\mathrm{CV}$ disease are unclear. Therefore, we investigated the relative contribution of traditional risk factors (such as smoking and hypertension), measures of inflammation associated with RA, and RA treatments to the increased risk of CV disease and mortality observed in RA.

In the Rochester RA cohort, a number of traditional risk factors for CV disease were found to contribute to $\mathrm{CV}$ death. These included a personal history of ischemic heart disease, smoking, hypertension, and diabetes mellitus. 15 Solomon et al30 reported an overall similar pattern of CV risk factors in a population of women with RA compared with women without RA. Our findings agree with these, in general, but we also observed some significant differences in the distribution of these risk factors between the 2 groups. ${ }^{18}$ During followup, RA patients were more likely to have low body mass index (BMI) (rate ratio [RR], 1.8; 95\% CI, 1.3-2.5) and alcohol abuse (RR, 3.3; 95\% CI, 1.7-7.6) but less likely to have dyslipidemia (RR, 0.8; 95\% CI, 0.6-0.9). In addition, the CV risk factors in RA patients did not appear sufficient to explain the increased risk of CV death in RA patients. The proportion of the risk of congestive heart failure that could be attributed to $\mathrm{CV}$ risk factors and ischemic heart disease was significantly lower in RA patients than in non-RA patients $(54 \%$ vs $77 \%$ at an age of $80 \mathrm{y}) .{ }^{13}$

Systemic inflammation associated with rheumatoid disease appears to account for a large proportion of this unexplained risk of $\mathrm{CV}$ death in the RA population. After adjustment for demographics, traditional $\mathrm{CV}$ risk factors, and comorbidities, we found that higher erythrocyte sedimentation rate (ESR), small and large joint swelling, rheumatoid nodules, vasculitis, and RA lung disease were all independently associated with an increased risk of CV death.15 In RA patients who experienced new-onset heart failure, the proportion of patients with very high ESR values $(\geq 40 \mathrm{~mm} / \mathrm{h}$ ) was greatest in the 6 months immediately preceding heart failure.31 These findings suggest that ESR, a marker of systemic inflammation, signals the onset of heart failure in persons with RA. These results are consistent with previous reports that have demonstrated an association between markers of 
systemic inflammation (including joint swelling32 and ESR33) and the risk of mortality in RA.

Assessment of the effect of RA medications on CV outcomes is complicated by the potential for confounding by indications and contraindications of the respective treatments. Therefore, their contribution to CV mortality is controversial. It has been suggested that treatment with corticosteroids may promote premature atherosclerosis and CV disease in RA patients.34 In our RA cohort, we observed an increased risk of CV events in RF+ RA patients with high cumulative exposure to corticosteroids (>7000 mg; HR, 3.06; 95\% CI, 1.81-5.18). ${ }^{35} \mathrm{In}$ contrast, RF- RA patients with high cumulative exposure to corticosteroids were not at increased risk of CV events (HR, 0.85; 95\% CI, 0.39-1.87). However, in RA patients with a personal history of ischemic heart disease, treatment with corticosteroids was found to attenuate the risk of $\mathrm{CV}$ death. 15

Use of methotrexate may also alter the risk of CV death in RA, although the nature of the effect is, at present, unclear. In one frequently cited study, use of methotrexate was found to be associated with a reduced risk of CV death (HR, 0.3; 95\% CI, 0.2-0.7), after adjustment for indication. ${ }^{36}$ Consistent with these data, treatment with methotrexate has also been associated with reduced vascular disease in RA patients (HR, 0.83; 95\% CI, 0.71-0.96).37 However, in another study using similar analysis techniques, use of methotrexate in patients with CV disease was associated with marked and significant increase in mortality (HR, 3.40; $P=0.0054) .{ }^{38}$ Thus,more research is needed to fully understand the impact of disease modifying anti-rheumatic drugs on CV risk. ${ }^{39,40}$

\section{Conclusions}

In summary, we observed a significantly increased risk of overall mortality, CV death, ischemic heart disease, and heart failure compared with the general population in a population-based incidence cohort of RA patients followed up for approximately 15 years. The overall mortality gap between RA patients and the general population is not closing. Indeed, it appears to be widening. In addition to the increased prevalence of CV mortality in the RA population, the pattern of $\mathrm{CV}$ disease in this population appears to be different from that observed in the general population. RA patients are more likely to have silent ischemic heart disease and experience sudden death. They are also more likely to develop inflammation and unstable atherosclerotic plaques in their coronary arteries. Furthermore, patients with RA are not only at a high risk of developing heart failure, but are more likely to die shortly after heart failure and are more likely to experience heart failure with a preserved ejection fraction.

Increased attention to $\mathrm{CV}$ risk in RA will be necessary to reduce the excess $\mathrm{CV}$ mortality and morbidity in RA patients. Traditional CV risk factors make an important contribution to CV morbidity in RA and, as in the general population, efforts should be made to control these risk factors. However, it appears that the excess risk that is observed in the RA population can be explained, in part, by promotion of $\mathrm{CV}$ disease through increased systemic inflammation associated with RA. Therefore, aggressive efforts at traditional CV risk factor reduction combined with improved control of RA disease may offer the best chance of improving CV outcomes for RA patients. In particular, the deleterious effects of systemic inflammation might be reduced by more effective ("tighter") control, in the same way that tight control of diabetes mellitus is recommended to reduce the risk of CV disease. 41,42 Large trials examining the effect of tight control of inflammation on CV outcomes in RA will be necessary to answer this question. Further research also is likely to focus on the identification of high-risk groups for targeted preventive interventions. 


\section{Acknowledgments}

The author would like to thank Ali Hassan, PhD, for assistance in drafting this manuscript. NIH Grant support from R01 AR46849 and AR30582.

\section{References}

1. Watson DJ, Rhodes T, Guess HA. All-cause mortality and vascular events among patients with rheumatoid arthritis, osteoarthritis, or no arthritis in the UK General Practice Research Database. J Rheumatol 2003;30:1196-1202. [PubMed: 12784389]

2. Kvalvik AG, Jones MA, Symmons DP. Mortality in a cohort of Norwegian patients with rheumatoid arthritis followed from 1977 to 1992. Scand J Rheumatol 2000;29:29-37. [PubMed: 10722255]

3. Symmons DP, Jones MA, Scott DL, et al. Longterm mortality outcome in patients with rheumatoid arthritis: early presenters continue to do well. J Rheumatol 1998;25:1072-1077. [PubMed: 9632066]

4. Anderson ST. Mortality in rheumatoid arthritis: do age and gender make a difference? Semin Arthritis Rheum 1996;25:291-296. [PubMed: 8778985]

5. Cobb S, Anderson F, Bauer W. Length of life and cause of death in rheumatoid arthritis. N Engl J Med 1953;249:553-556. [PubMed: 13087647]

6. Myllykangas-Luosujarvi RA, Aho K, Isomaki HA. Mortality in rheumatoid arthritis. Semin Arthritis Rheum 1995;25:193-202. [PubMed: 8650589]

7. Gonzalez-Gay MA, Gonzalez-Juanatey C, Miranda-Filloy JA, et al. Cardiovascular disease in rheumatoid arthritis. Biomedicine and Pharmacotherapy 2006;60:673-677.

8. Solomon DH, Goodson NJ, Katz JN, et al. Patterns of cardiovascular risk in rheumatoid arthritis. Ann Rheum Dis 2006;65:1608-1612. [PubMed: 16793844]

9. Maradit-Kremers H, Crowson CS, Nicola PJ, et al. Increased unrecognized coronary heart disease and sudden deaths in rheumatoid arthritis: a population-based cohort study. Arthritis Rheum 2005;52:402-411. [PubMed: 15693010]

10. Libby P. Inflammation and cardiovascular disease mechanisms. Am J Clin Nutr 2006;83:456S460S. [PubMed: 16470012]

11. Melton LJ 3rd. History of the Rochester Epidemiology Project. Mayo Clin Proc 1996;71:266-274. [PubMed: 8594285]

12. Nicola PJ, Crowson CS, Maradit-Kremers H, et al. Contribution of congestive heart failure and ischemic heart disease to excess mortality in rheumatoid arthritis. Arthritis Rheum 2006;54:60-67. [PubMed: 16385496]

13. Crowson CS, Nicola PJ, Kremers HM, et al. How much of the increased incidence of heart failure in rheumatoid arthritis is attributable to traditional cardiovascular risk factors and ischemic heart disease? Arthritis Rheum 2005;52:3039-3044. [PubMed: 16200583]

14. Gabriel SE, Crowson CS, Kremers HM, et al. Survival in rheumatoid arthritis: a population-based analysis of trends over 40 years. Arthritis Rheum 2003;48:54-58. [PubMed: 12528103]

15. Maradit-Kremers H, Nicola PJ, Crowson CS, et al. Cardiovascular death in rheumatoid arthritis: a population-based study. Arthritis Rheum 2005;52:722-732. [PubMed: 15751097]

16. Wallberg-Jonsson S, Ohman ML, Dahlqvist SR. Cardiovascular morbidity and mortality in patients with seropositive rheumatoid arthritis in Northern Sweden. J Rheumatol 1997;24:445-451. [PubMed: 9058647]

17. Goodson NJ, Wiles NJ, Lunt M, et al. Mortality in early inflammatory polyarthritis: cardiovascular mortality is increased in seropositive patients. Arthritis Rheum 2002;46:2010-2019. [PubMed: 12209502]

18. Gonzalez A, Maradit-Kremers H, Crowson CS, et al. The incidence of cardiovascular risk factors in rheumatoid arthritis [abstract]. Arthritis Rheum 2005;54:S785.

19. Wolfe F, Freundlich B, Straus WL. Increase in cardiovascular and cerebrovascular disease prevalence in rheumatoid arthritis. J Rheumatol 2003;30:36-40. [PubMed: 12508387]

20. Solomon DH, Karlson EW, Rimm EB, et al. Cardiovascular morbidity and mortality in women diagnosed with rheumatoid arthritis. Circulation 2003;107:1303-1307. [PubMed: 12628952] 
21. Fischer LM, Schlienger RG, Matter C, et al. Effect of rheumatoid arthritis or systemic lupus erythematosus on the risk of first-time acute myocardial infarction. Am J Cardiol 2004;93:198200. [PubMed: 14715346]

22. Turesson C, Jarenros A, Jacobsson L. Increased incidence of cardiovascular disease in patients with rheumatoid arthritis: results from a community based study. Ann Rheum Dis 2004;63:952955. [PubMed: 15051620]

23. Sodergren A, Stegmayr B, Lundberg V, et al. Increased incidence of and impaired prognosis after acute myocardial infarction among patients with seropositive rheumatoid arthritis. Ann Rheum Dis 2007;66:263-266. [PubMed: 16854951]

24. Aubry MC, Maradit-Kremers H, Reinalda MS, et al. Differences in Atherosclerotic Coronary Heart Disease Between Subjects with and without Rheumatoid Arthritis. J Rheumatol. 2007

25. Gabriel SE, Crowson CS, O'Fallon WM. Comorbidity in arthritis. J Rheumatol 1999;26:24752479. [PubMed: 10555912]

26. Wolfe F, Michaud K. Heart failure in rheumatoid arthritis: rates, predictors, and the effect of antitumor necrosis factor therapy. Am J Med 2004;116:305-311. [PubMed: 14984815]

27. Nicola PJ, Maradit-Kremers H, Roger VL, et al. The risk of congestive heart failure in rheumatoid arthritis: a population-based study over 46 years. Arthritis Rheum 2005;52:412-420. [PubMed: 15692992]

28. Davis JM 3rd, Crowson CS, Maradit-Kremers H, et al. Heart failure occurs more frequently with a preserved ejection fraction in subjects with rheumatoid arthritis (RA) compared to non-RA subjects [abstract]. Arthritis Rheum 2006;54:S388.

29. Davis JM 3rd, Crowson CS, Maradit-Kremers H, et al. Mortality following heart failure is higher among rheumatoid arthritis (RA) subjects compared to non-RA subjects [abstract]. Arthritis Rheum 2006;54:S387-S388.

30. Solomon DH, Curhan GC, Rimm EB, et al. Cardiovascular risk factors in women with and without rheumatoid arthritis. Arthritis Rheum 2004;50:3444-3449. [PubMed: 15529391]

31. Maradit-Kremers H, Nicola PJ, Crowson CS, et al. Raised erythrocyte sedimentation rate signals heart failure in patients with rheumatoid arthritis. Ann Rheum Dis 2007;66:76-80. [PubMed: 16818462]

32. Jacobsson LT, Turesson C, Hanson RL, et al. Joint swelling as a predictor of death from cardiovascular disease in a population study of Pima Indians. Arthritis Rheum 2001;44:11701176. [PubMed: 11352251]

33. Wallberg-Jonsson S, Johansson H, Ohman ML, et al. Extent of inflammation predicts cardiovascular disease and overall mortality in seropositive rheumatoid arthritis. A retrospective cohort study from disease onset. J Rheumatol 1999;26:2562-2571. [PubMed: 10606363]

34. Moreland LW, O'Dell JR. Glucocorticoids and rheumatoid arthritis: back to the future? Arthritis Rheum 2002;46:2553-2563. [PubMed: 12384910]

35. Davis JM 3rd, Maradit Kremers H, Crowson CS, et al. Glucocorticoids and cardiovascular events in rheumatoid arthritis: A population-based cohort study. Arthritis Rheum 2007;56:820-830. [PubMed: 17330254]

36. Choi HK, Hernan MA, Seeger JD, et al. Methotrexate and mortality in patients with rheumatoid arthritis: a prospective study. Lancet 2002;359:1173-1177. [PubMed: 11955534]

37. Prodanovich S, Kirsner RS, Taylor JR. Treatment of patients hospitalized for psoriasis. Dermatol Clin 2000;18:425-435. viii. [PubMed: 10943538]

38. Landewe RB, van den Borne BE, Breedveld FC, et al. Methotrexate effects in patients with rheumatoid arthritis with cardiovascular comorbidity. Lancet 2000;355:1616-1617. [PubMed: 10821370]

39. Suissa S, Bernatsky S, Hudson M. Antirheumatic drug use and the risk of acute myocardial infarction. Arthritis Rheum 2006;55:531-536. [PubMed: 16874796]

40. van Halm VP, Nurmohamed MT, Twisk JW, et al. Disease-modifying antirheumatic drugs are associated with a reduced risk for cardiovascular disease in patients with rheumatoid arthritis: a case control study. Arthritis Res Ther 2006;8:R151. [PubMed: 16984661]

41. Basile JN. Optimizing antihypertensive treatment in clinical practice. Am J Hypertens 2003;16:13S-17S. [PubMed: 14511897] 
42. Pincus T, Gibofsky A, Weinblatt ME. Urgent care and tight control of rheumatoid arthritis as in diabetes and hypertension: better treatments but a shortage of rheumatologists. Arthritis Rheum 2002;46:851-854. [PubMed: 11953958] 


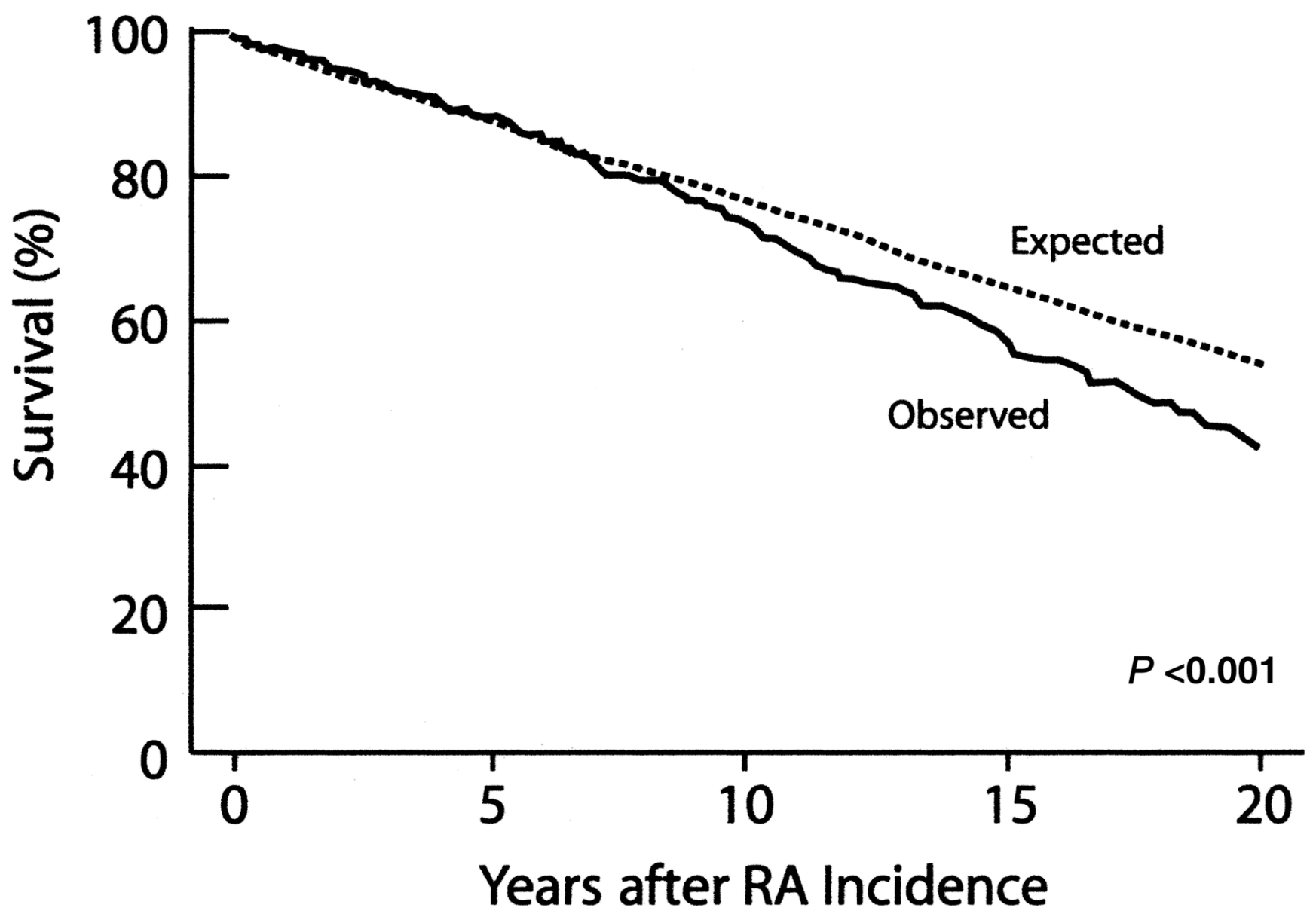

Figure 1.

Increased mortality in patients with rheumatoid arthritis (RA). Adapted with permission from Gabriel SE, Crowson CS, Kremers HM, et al. Survival in rheumatoid arthritis: a population-based analysis of trends over 40 years. Arthritis Rheum. 2003;48:54-58. 


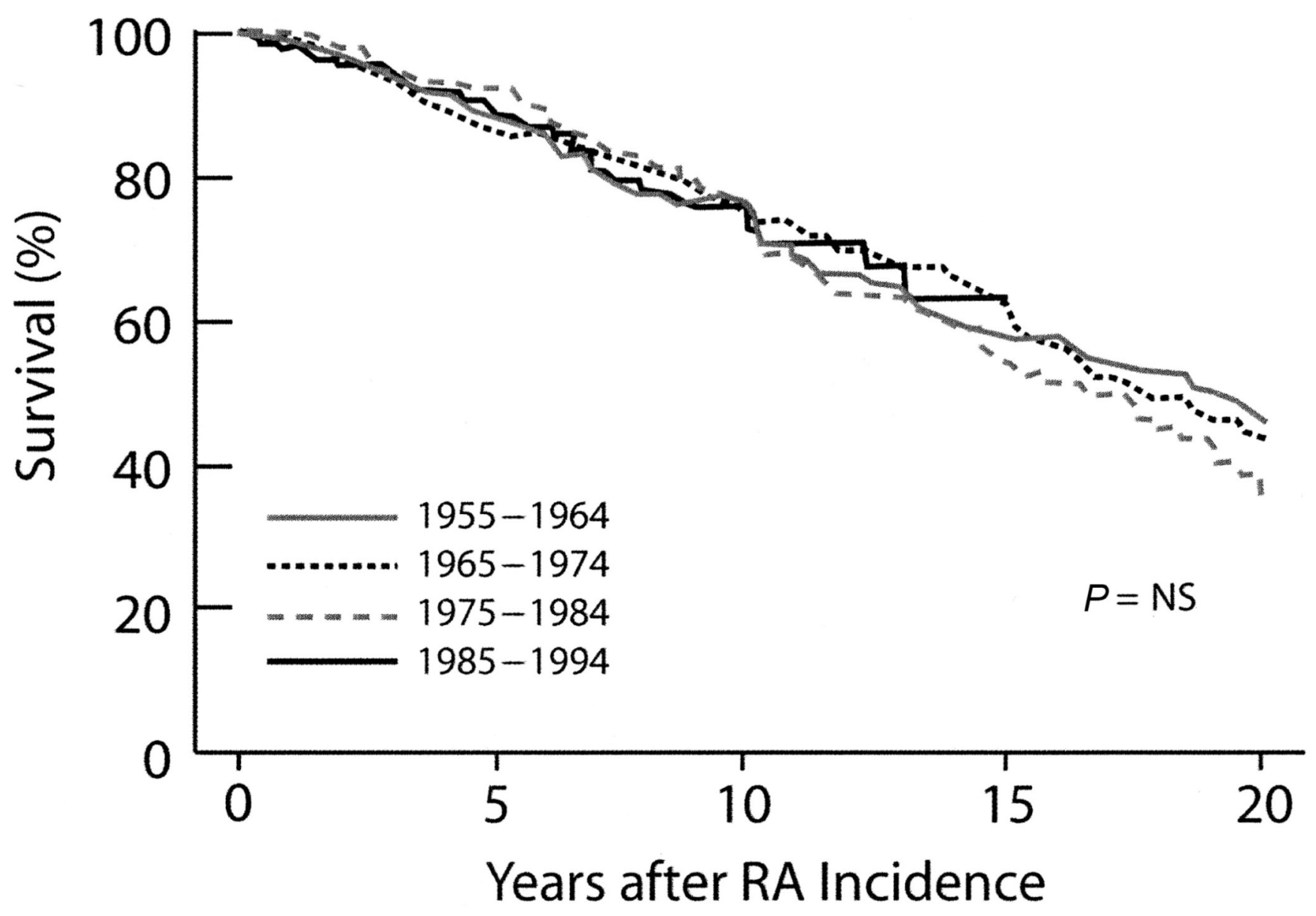

Figure 2.

Survival among 4 cohorts of rheumatoid arthritis (RA) patients first diagnosed between 1955 and 1995. Adapted with permission from Gabriel SE, Crowson CS, Kremers HM, et al. Survival in rheumatoid arthritis: a population-based analysis of trends over 40 years. Arthritis Rheum. 2003;48:54-58. 


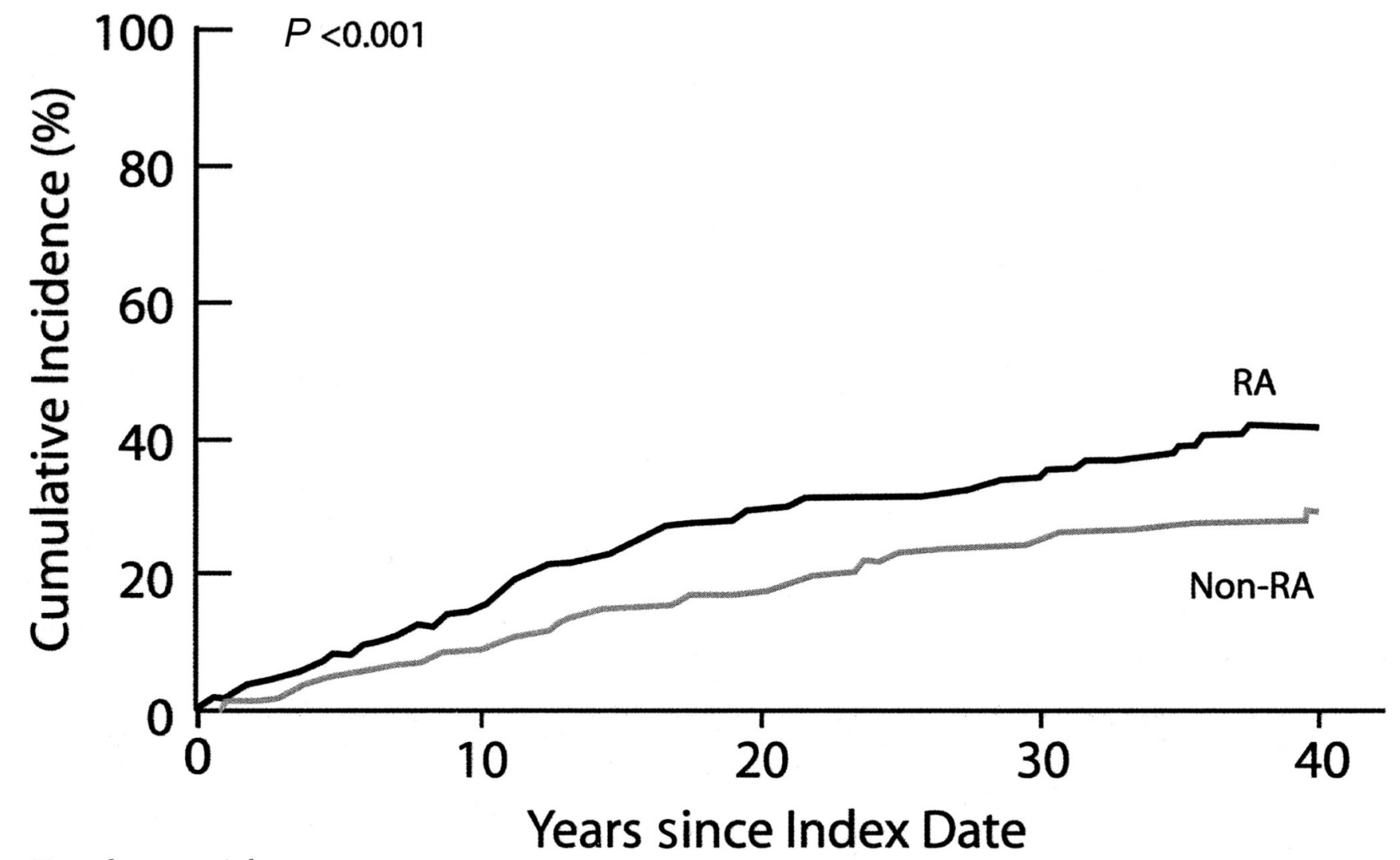

Number at risk

$\begin{array}{rlccc}\text { RA } & 575 & 336 & 133 & 51 \\ \text { Non-RA } & 583 & 386 & 189 & 75 \\ & & & \\ & \begin{array}{l}\text { Figure 3. } \\ \text { Cumulative incidence of congestive heart failure in rheumatoid arthritis (RA) and non-RA } \\ \text { patients. Adapted with permission from Nicola PJ, Maradit-Kremers H, Roger VL, et al. The } \\ \text { risk of congestive heart failure in rheumatoid arthritis: a population-based study over } 46 \\ \text { years. Arthritis Rheum. 2005;52:412-420. }\end{array}\end{array}$




\section{Table}

Risk of Ischemic Heart Disease in RA and Non-RA Patients After the Incidence of Rheumatoid Arthritis

\begin{tabular}{|c|c|c|c|}
\hline & \multicolumn{2}{|c|}{ Number of Events (Rate/10,000 Person-years) } & \multirow{2}{*}{$\begin{array}{l}\text { Multivariable-Adjusted } \\
\text { Hazard Ratio } \\
\text { (95\% Confidence Interval) }\end{array}$} \\
\hline & RA Cohort & Non-RA Cohort & \\
\hline Hospitalized MI & $40(46)$ & $46(46)$ & $1.09(0.71-1.68)$ \\
\hline Unrecognized MI & $27(31)$ & $15(15)$ & $2.13(1.13-4.03)$ \\
\hline Angina pectoris & $44(51)$ & $65(67)$ & $0.76(0.52-1.13)$ \\
\hline Revascularization procedure & $28(32)$ & $35(35)$ & $0.94(0.56-1.56)$ \\
\hline Sudden death & $31(35)$ & $18(18)$ & $1.94(1.06-3.55)$ \\
\hline
\end{tabular}

$\mathrm{MI}=$ myocardial infarction .

Adapted with permission from Maradit-Kremers H, Crowson CS, Nicola PJ, et al. Increased unrecognized coronary heart disease and sudden deaths in rheumatoid arthritis: a population-based cohort study. Arthritis Rheum. 2005a;52:402-411. 